\title{
Spectral Analysis of Ultrasonic and Photo Acoustic Signals Generated by a Prototypal Fiber Microprobe for Media Characterization
}

\author{
Simona Granchi ${ }^{1}$ (D) Enrico Vannacci ${ }^{1}$ - Lorenzo Miris ${ }^{1} \cdot$ Luca Onofri $^{1}$. \\ Duccio Zingoni ${ }^{1}$ - Elena Biagi ${ }^{1}$
}

Received: 15 April 2019 / Revised: 13 April 2020 / Published online: 9 July 2020

(C) The Author(s) 2020, corrected publication 2020

\begin{abstract}
Combination of photoacoustics and ultrasound can provide complemental features and mutual benefits, useful for a complete tissue characterization and consequently for early diagnosis or therapy monitoring. Furthermore, minimally invasive techniques are required both to reach organs or tissue not accessible and to reduce patient discomfort and costs. This work has tested a prototypal microprobe for media characterization analysing their optical and mechanical features. Two different transmitters compose the miniaturized probe: one for large bandwidth ultrasonic signals generation and one for guiding the laser light into tissue to photogenerate ultrasound. The aim is to evaluate the possibility of employing in the future this new type of microprobe to characterize internal tissue, combining ultrasound and photoacoustic investigations. A calibrated commercial hydrophone has been used to detect generated signals, with the aim to provide repeatable and reliable results. Dedicated test objects have been realized by using solutions of corn starch flour and of Chinese ink with different and calibrated dilutions. The spectral algorithm HyperSPACE (Hyper SPectral Analysis for Characterization in Echography), applied on ultrasonic and photoacoustic signals has allowed differentiating scatterers' concentration and distribution.
\end{abstract}

Keywords Photoacoustics $\cdot$ Ultrasound $\cdot$ Spectral processing $\cdot$ Media characterization $\cdot$ Fully fiber microprobe $\cdot$ Minimally invasiveness

Simona Granchi

simona.granchi@unifi.it

1 Ultrasound and Non-Destructive Testing Lab, Department of Information Engineering (DINFO), University of Florence, via Santa Marta 3, 50139 Florence, Italy 


\section{Introduction}

The phenomenon of photoacoustic effect has been known for many years, but its investigation and application had to wait until the development of proper laser sources, sensitive and broadband ultrasound detection technologies, fast data acquisition and processing capabilities [1]. The peculiar and effective characteristic of photoacoustics is the combination of the advantages of optical techniques, such as the high resolution and of some features of ultrasound, such as penetration into materials [2, 3]. The first application of this technique was non-destructive testing (NDT) of mechanical and elastic properties of materials, but, since the 1960s [1], the interest in biomedical field began to grow. In the last 2 decades, the biomedical optoacoustic research has oriented to develop suitable detectors and processing techniques [1, 4-12], in order to produce images able to characterize healthy and pathological tissue. Important works have proven that photoacoustic imaging is capable to provide information on tissue conditions [5, 13-20], so as to represent a potential tool for improving the early clinical diagnosis and for monitoring therapeutic operations [21-24]. Moreover, one of the trending topics is the multiple imaging technique, which combines photoacoustics (PA) and ultrasound (US), providing complementary features [15, 24-31]. The US images are able to represent the tissue morphological, dimensional and mechanical characteristics, but the low acoustic contrast between soft tissues prevents to characterize their specific composition in detail, although important progresses have been performed in signal and imaging processings and in technologies [23, 32, 33]. On the other hand, PA images allow to differentiate the tissue according to the optical absorption, so that they distinguish an atherosclerotic plaque into a carotid vessel $[8,15]$ or detect cancerous formations from the surrounding tissue $[34,35]$. Moreover tissue imaging presents diagnostic capability if it exhibits high resolution and so large spectral bandwidth transducers are required, but, as known, the US penetration is hardly limited at high frequencies. Many efforts and technological solutions have been developed for suitable optical and ultrasonic sensors [12, 36-44] and also our research group has been working for several years on a micro fiber probe [45-51]. In this probe, ultrasound generation occurs though thermoelastic emission from patterned carbon films in micro-optomechanical-system (MOMS) devices mounted on the tip of an optical fiber. The miniaturization allows it to be driven via catheters or endoscopes into the natural orifices of the human body to reach the organ under investigation in a percutaneous manner, using a needle of a syringe as a guide. Moreover, the high resolution of the microprobe may enable a minimally invasive "virtual biopsy" of internal tissue or organs.

In the present work, we present an experimental method to test the capabilities of a prototypal microprobe to characterize two simple test objects as function of their optical and mechanical features. The aim is to evaluate the possibility of employing in the future this new type of microprobe to characterize internal tissue, combining US and PA investigations.

Indeed, the miniaturized ultrasonic transmitter has been used in conjunction with an optical fiber to drive the laser light into the tissue for PA generation. 
Moreover, we have used a commercial hydrophone to collect both ultrasonic, and photoacoustic signals.

In order to control the mechanical and optical features, we have made two simple test objects, where only one parameter changed: the dilution. Test objects consisted of corn starch flour and Chinese ink at different concentration levels. Some authors have proven [52] that the spectral analysis of the radiofrequency photoacoustic signals may improve the characterization of absorbers' size and concentration and also in this work we have performed a spectral processing on both PA and US radiofrequency signals. By applying the hyperspectral method HyperSPACE (Hyper SPectral Analysis for Characterization in Echography) presented by authors in previous works [53-55], we have studied how the spectral features of PA and US signals may characterize the different media at different dilutions. In clinical practice, a device able to reach internal organs or tissue has to meet the requirement of assuring the minimal invasiveness for patient. Consequently, a microprobe has to be compact by including transmitters and receivers in the same device. In this field, the authors are studying solutions to realize a fully fiber microprobe containing optical, ultrasonic transmitters and receivers.

\section{Materials and Methods}

\subsection{Experimental Set Up}

The measurement chain is reported in Fig. 1. The experiments include the microprobe composed by the MOMS ultrasonic transmitter described in detail in [46-50], for ultrasonic signal (US) generation and an optical fiber transporting light, for photoacoustic signal (PA) generation in the investigated medium.

The US transmitter has dimensions (roughly $350 \mu \mathrm{m} \times 220 \mu \mathrm{m}$ ) small enough to be inserted into a syringe needle once mounted onto the optical fibers. The spectrum of US signal, measured in [48], is rather flat and reaches very high frequencies. A Q-switch 1064 nm Nd:YAG laser (Teem Photonics, PNP-005025-140) has been used to feed the optoacoustic MOMS source mounted on a multi-mode optical fiber (230 $\mu \mathrm{m}$ diameter) with a pulse duration of $500 \mathrm{ps}$ and a pulse repetition frequency of $1 \mathrm{kHz}$.

The microprobe, reported in Fig. 2 has been used in echo configuration and the HGL-0200 hydrophone (Onda Corporation, USA) has been used as receiver. Its nominal sensitivity is of $40 \mathrm{nV} / \mathrm{Pa}$ and has $\mathrm{a} \pm 3 \mathrm{~dB}$ bandwidth between 0.25 and $40 \mathrm{MHz}$. A digital oscilloscope (TDS model, Tektronix Company, USA) has been employed to acquire both US and PA radiofrequency (RF) signals while a signal, provided by laser, was used as trigger.

Demineralized water has been used as the medium for coupling microprobe to test object and as solvent for the analysed solutions. Test objects have been developed in order to have a scatterers' distributions with different optical and mechanical properties, able to simulate most of soft tissue [56] and in order to exhibit complementary acoustic and optical characteristics. Chinese-ink and corn starch flour met these requirements. Indeed, the first one, presents high optical absorption associated 


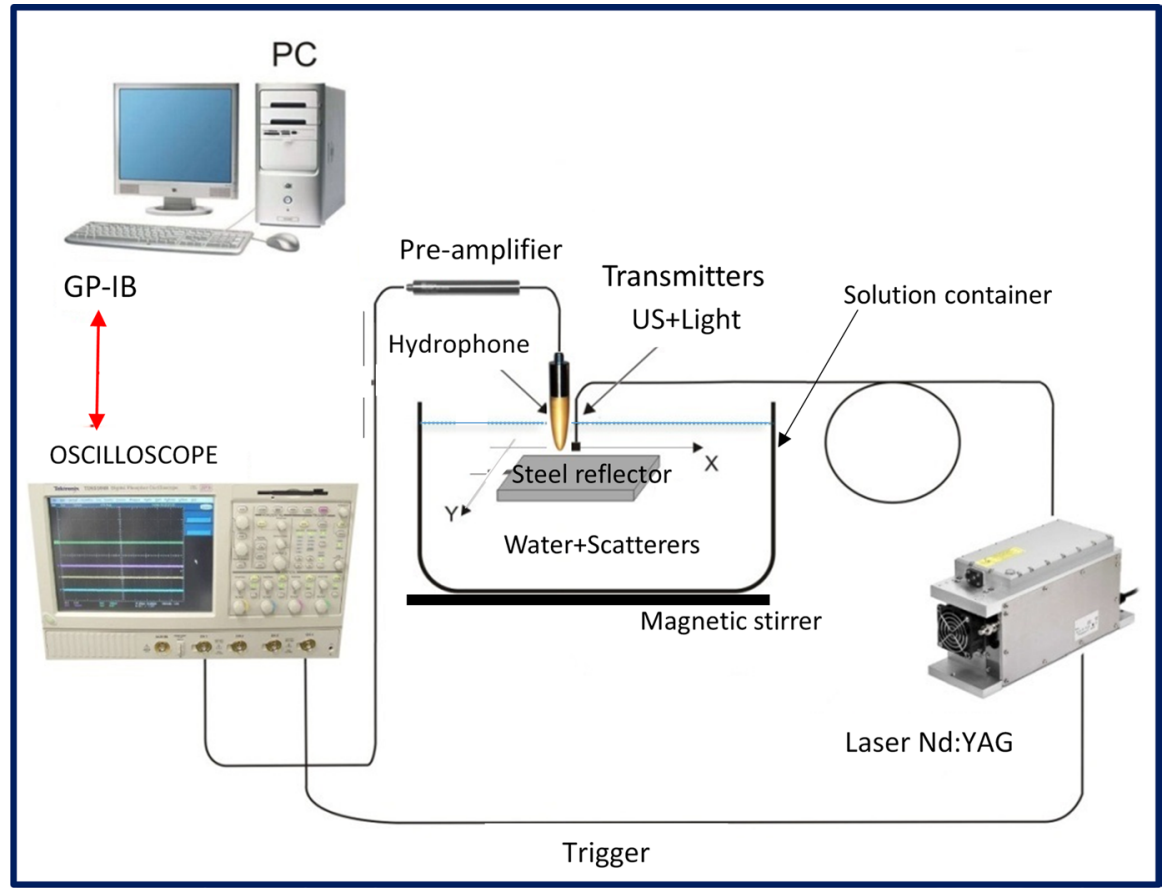

Fig. 1 Measurement set up for US and PA signals acquisition. Laser Nd:YAG provides light both to a MOMS ultrasonic transmitter and to an optical fiber for light transport (transmitters US + light). The laser light induces PA generation into the test object while the opto acoustic transmitter, included in the same microprobe, produces US, which travel into the test object. The PA and US signals, back propagated by test object, are collected by the hydrophone and saved though the Oscilloscope. Through the GP-IB line, data are transferred in the PC and processed

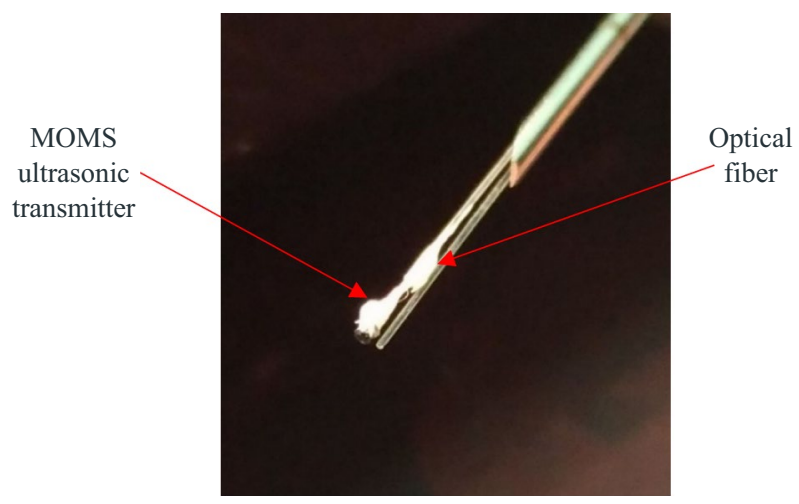

Fig. 2 Photo of realized microprobe constituted by MOMS ultrasonic transmitter and an optical fiber for light transport 
to a low acoustic attenuation coefficient and the second, high acoustic attenuation coefficient associated to low optical absorption. Moreover, these substances are easily available. Experimentation has been performed in order to analyze and characterize two test objects composed by two different solutions: the first containing Chinese ink (mean diameter $D_{\mathrm{C}} \approx 200 \mathrm{~nm}$ ) and the second, corn starch flour (mean diameter $D_{\mathrm{m}} \approx 5 \mu \mathrm{m}$ ), at different dilutions. The concentration has been systematically changed by adding a calibrated quantity of substance to the solution, which has been continuously mixed through an magnetic stirrer (Velp Scientifica, Milan, Italy), in order to maintain the homogeneity, preventing accumulation and sedimentation of the medium that would have led to unpredictable local changes in its concentration, especially in the proximity of the probes. The initial solution was only water $(600 \mathrm{ml})$. Transmitters and the hydrophone were immersed into a container with the solution inside, as shown in Fig. 3.

66 solutions of Chinese ink with a dilution range of $[0,0.4] \mathrm{ml} / \mathrm{ml}$ and 66 corn starch flour solutions with a dilution range of $[0,0.1] \mathrm{g} / \mathrm{ml}$ have been analyzed; for each dilution level, 64 signals have been acquired and processed to extract the average signal, in order to improve the signal to noise ratio (SNR). Time algorithms have been applied to extract peak-to-peak values of the US and PA signals respectively, in order to evaluate the medium attenuation as function of dilution. Moreover, spectral processing was used to identify the parameters sensitive to dilution variations; in particular, the HyperSPACE coefficients [53-55] have been studied. It is worth to note that this kind of analysis reveals the optical and mechanical characteristics of the medium even if the signal amplitude is very low, confirming that HyperSPACE algorithm results are not affected by signal amplitude.

\section{Results}

Experiments for test objects characterization have been performed by following the dilution procedures reported in Table 1 for the Chinese ink and in Table 2 for the corn starch flour.

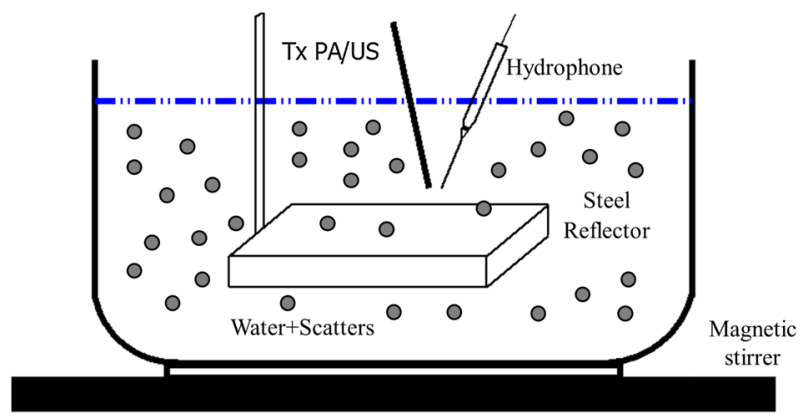

Fig. 3 Test object constituted by solution of water and scatterers of corn starch or Chinese ink. Microprobe and hydrophone are in echo configuration. A magnetic stirrer assures homogeneity of the solution during addition of the medium 
Table 1 Dilution procedure for changing concentration of Chinese ink solution

\begin{tabular}{llll}
\hline $\begin{array}{l}\text { Acquisition } \\
\text { numbers }\end{array}$ & $\begin{array}{l}\text { Addition for each } \\
\text { step dilution }(\mathrm{ml})\end{array}$ & $\begin{array}{l}\text { Subtotal addi- } \\
\text { tion }(\mathrm{ml})\end{array}$ & $\begin{array}{l}\text { Total volume } \\
\text { solution }(\mathrm{ml})\end{array}$ \\
\hline $0-8$ & 0.08 & 0.7 & 600.7 \\
$9-14$ & 0.2 & 1.2 & 601.9 \\
$15-22$ & 0.4 & 3.2 & 605.0 \\
$23-31$ & 5 & 45 & 650.0 \\
$32-66$ & 10 & 340 & 990.0 \\
\hline
\end{tabular}

\begin{tabular}{lllll}
\cline { 2 - 5 } $\begin{array}{l}\text { Table 2 Dilution procedure for } \\
\text { changing concentration of corn } \\
\text { starch flour solution }\end{array}$ & $\begin{array}{l}\text { Acquisition } \\
\text { numbers }\end{array}$ & $\begin{array}{l}\text { Addition for each } \\
\text { step dilution }(\mathrm{ml})\end{array}$ & $\begin{array}{l}\text { Subtotal addi- } \\
\text { tion (ml) }\end{array}$ & $\begin{array}{l}\text { Total volume } \\
\text { solution (ml) }\end{array}$ \\
\cline { 2 - 5 } & $0-25$ & 0.2 & 5 & 605.0 \\
& $26-38$ & 1 & 13 & 618.0 \\
& $39-56$ & 5 & 90 & 708.0 \\
& $57-66$ & 5 & 50 & 758.0 \\
\hline
\end{tabular}

Table 2 Dilution procedure for changing concentration of corn starch flour solution
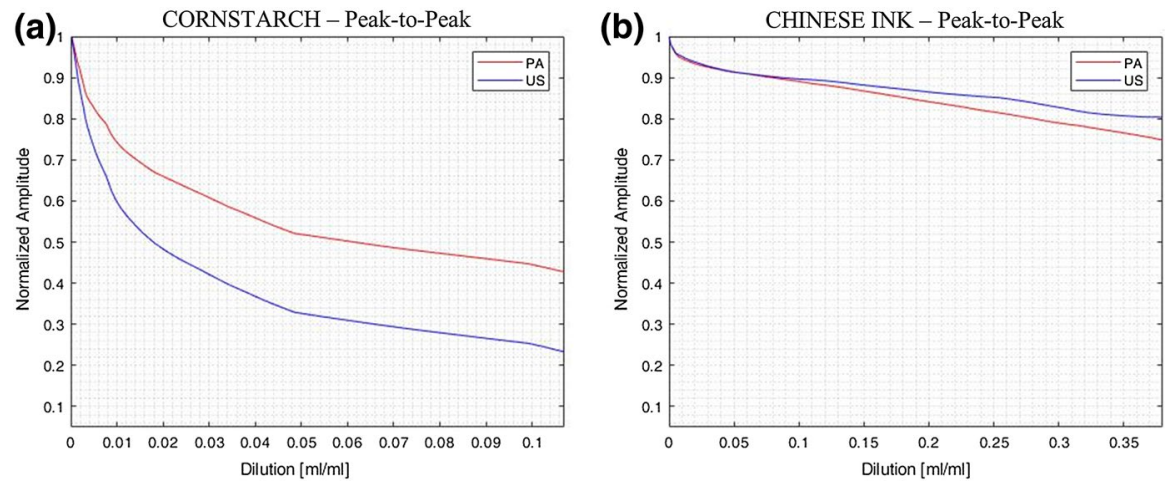

Fig. 4 Peak to peak values of the PA (red) and US (blue) signals as function of dilution for of corn starch (a) and Chinese ink (b). The values are normalized in respect of the corresponding value of initial dilution (only water) (Color figure online)

Both US signals, reflected by a steel interface, placed at the bottom of a container with solution inside (Fig. 3) and the PA signals photo generated by the same, have been analyzed to estimate the changes occurred as dilution changed.

Moreover, the curves of Fig. 5 also confirm this different behavior. They represent the mean frequency shift as function of dilution, in respect of initial condition.

US and PA signals, referred to each acquisition, have been processed in time and frequency domains. Peak-to peak values and frequency mean values as functions of dilution have been estimated and represented in the diagrams reported in Figs. 4 and 5. The first diagram of Fig. 4a, relative to corn starch flour, shows two curves: one 

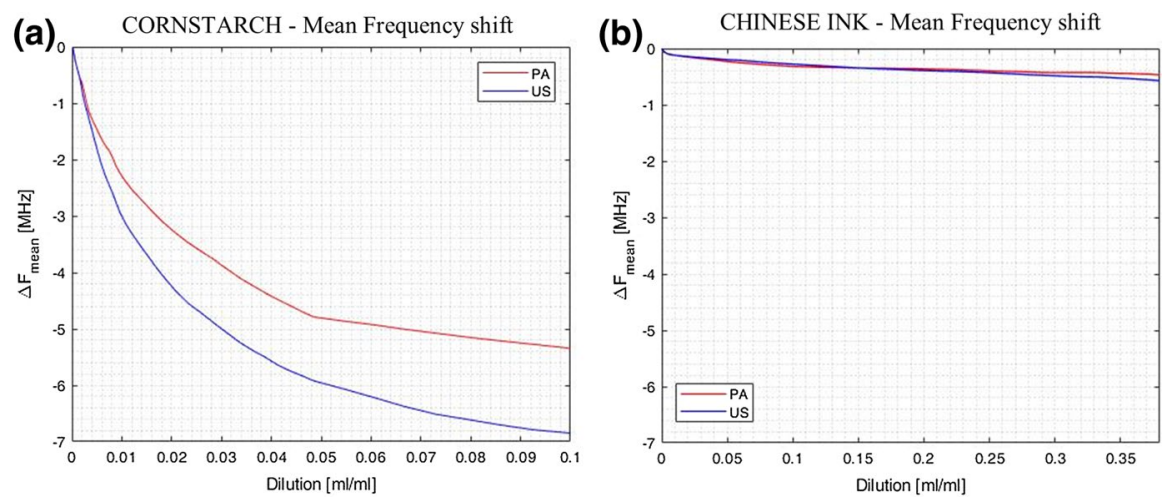

Fig. 5 Mean frequency shift of the PA (red) and US (blue) signals, in respect of the initial mean frequency values as function of dilution, for the corn starch (a) and Chinese ink (b) (Color figure online)

red for PA signals and one blue for the US. They represent the peak-to-peak values for each dilution, normalized in respect of the initial value (solution with only water). Instead, Fig. 4b represents Chinese ink. The variations of peak-to peak values of US signal at different dilution steps are different in respect of those of PA signal, as result of the different interaction of the two substances with optical and ultrasonic waves.

The method HyperSPACE has been applied on both PA and US signals, after having decomposed the spectra into 40 bands of $2 \mathrm{MHz}$ bandwidth and with an overlap of $1 \mathrm{MHz}$ in a frequency range of $2-40 \mathrm{MHz}$ by analyzing the HyperSPACE coefficients $h s[53,54]$ in the hyperspace, that has 40 dimensions [54], it is possible to detect different distributions according to the different dilutions, as shown in Figs. 6, 7. The $h s$ clusters for US (Figs. 6a, 7a) and for PA (Figs. 6b, 7b) of Chinese ink and of corn starch flour are respectively represented in a bidimensional projection of the hyperspace as function of dilution that increases as indicated by the arrow.
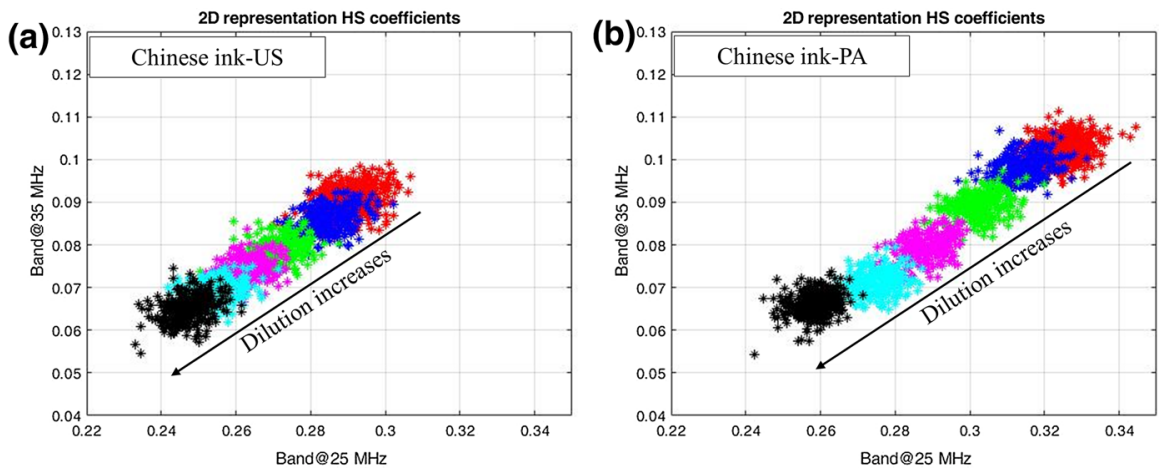

Fig. 6 Clusters distribution of HyperSpace coefficients (hs) as function of dilution, referred to US signal of Chinese ink in a and to PA signal of Chinese ink in $\mathbf{b}$. Each coloured cluster corresponds to a specific dilution (Color figure online) 

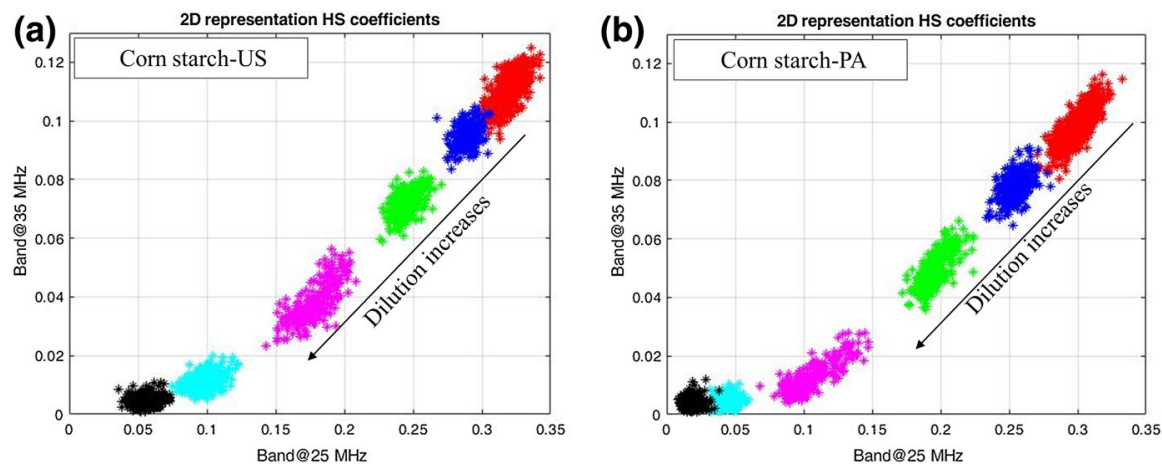

Fig. 7 Clusters distribution of HyperSpace coefficients (hs) as function of dilution referred to US signal of corn starch flour in a and to PA signal of corn starch in $\mathbf{b}$. Each colored cluster corresponds to a specific dilution (Color figure online)

\section{Discussion}

The simultaneous and complementary use of PA and US provides mechanical, structural and optical characteristics of the analysed medium. Moreover the PA signal overcomes the penetration limits of the light, preserving the advantages in a selective characterization of the medium, as shown in Figs. $4 \mathrm{~b}$ and 5b, where the signal is only generated and detected in correspondence of the wavelength absorbed by the investigated material (Chinese ink in this case). Measurements have produced multiple data (US and PA) able to provide different information from the analysed test objects, in terms of mechanical and structural features (dilution, and composition), as reported on the diagrams in Figs. 4, 5, 6, 7 and of optical behaviour, as it is inferable from the same diagrams. Indeed, both in corn starch flour and Chinese ink solutions, the photo generation occurs but in the first case, the particles have low optical absorption coefficient, while in the second one, the Chinese ink particles have a higher coefficient [57]. Consequently, in the corn starch solution, the predominant effect is the signal attenuation due to acoustical absorption and scattering, which increases as dilution becomes higher. The difference between the attenuation rate of the curves in Figs. 4a and 5a referred to PA and US signal respectively, depends on US and PA paths. In fact, the first is twice the second in length, as shown in the Fig. 8 and the attenuation suffered by the US signal is higher than that of PA.

Instead, in the Chinese ink solution, the optical absorption effect is considerable and prevents the light to penetrate in depth as the particles concentration increases. Therefore, globally, the PA signal decreases more than US, even if its path is half of the other. It is also possible to note that the attenuation of both signals in the Chinese ink solution is less than that in corn starch solution (Figs. 4, 5) and it is probably due to the different sizes of particles and their different chemical and structural composition [58]. Moreover, by exploiting the HyperSPACE method potentialities, media characterization can be improved, combining the results obtained by the two signals respectively. For example, in Fig. 6a) the $h s$ clusters of US signals, related to different dilutions, are partially overlapped, while those of PA signals, generated 
(a)

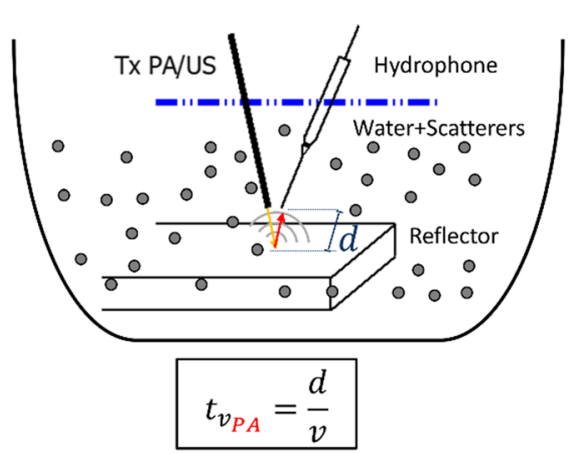

(b)

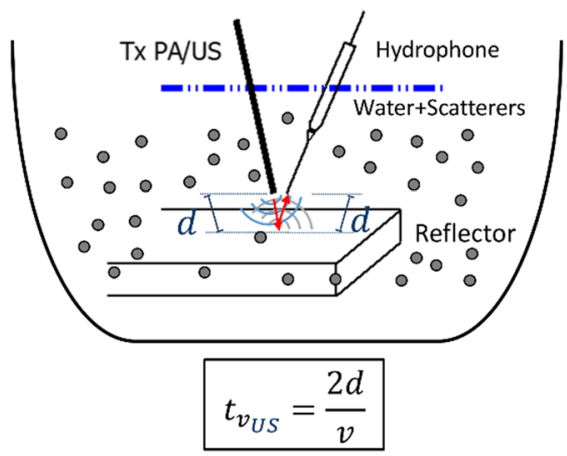

Fig. 8 Set up for ultrasonic (US) and photoacoustic (PA) signals detection. a PA generated by the steel reflector travels half path with respect of US in $\mathbf{b}$, reflected by the same target, before to reach the hydrophone

selectively according to the different concentration of ink scatterers, are more separated, providing a better dilutions discrimination. On the other hand, the combination of information obtained using different energy sources (light and mechanical waves) is a challenging frontier but not a novelty in this research field $[4,15$, 22, 24-27, 29-31, 39, 40, 58]. A new important technological improvement may be represented by the fully fiber microprobe, which our research group are working on. Indeed, microprobes, composed by US transducer and optical fiber as transmitters and acousto-optical fiber elements as receivers may satisfy the growing need for early diagnosis with minimal invasive impact in clinical field or the necessity of characterizing inaccessible structures by using internal probes in non-destructive testing field. Figure 9 shows an example of a possible prototype with a single receiver.

However, for clinical application, it is important to acquire and produce real time images of tissue in order to support both diagnosis and therapy monitoring, ensuring the minimal invasiveness of examination. A possible solution may be a US-PA microprobe constituted of one optical fiber for light transport into tissue, of one fiber optoacoustic transmitter and of multiple receivers mounted on a fiber bundle.

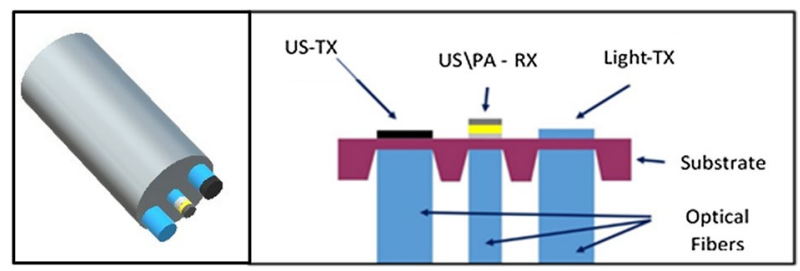

Fig. 9 A proposal of a fully fiber microprobe prototype, constituted of a MOMS US transmitter, an optical fiber for light transport and a acousto-optical fiber for US and PA reception 


\section{Conclusion}

A prototypal fully fiber transmitter, constituted by an optoacoustic transducer in MOMS technology, for large spectral bandwidth US generation coupled to an optical fiber, for PA generation, has been employed to conduct experiments on media characterization. In reception, commercial hydrophone has been used in order to provide repeatable and calibrated data. Results on solutions with corn starch flour and Chinese ink, having different optical properties and different concentrations have been presented. For clinical applications, the authors are developing a fully fiber microprobe by composing an optical fiber for light transport into tissue, an optoacoustic transmitter and a receiver with dimensions that may permit it to be inserted in needles or endoscopic cables.

Funding Open access funding provided by Università degli Studi di Firenze within the CRUI-CARE Agreement.

Open Access This article is licensed under a Creative Commons Attribution 4.0 International License, which permits use, sharing, adaptation, distribution and reproduction in any medium or format, as long as you give appropriate credit to the original author(s) and the source, provide a link to the Creative Commons licence, and indicate if changes were made. The images or other third party material in this article are included in the article's Creative Commons licence, unless indicated otherwise in a credit line to the material. If material is not included in the article's Creative Commons licence and your intended use is not permitted by statutory regulation or exceeds the permitted use, you will need to obtain permission directly from the copyright holder. To view a copy of this licence, visit http://creativecommons.org/licen ses/by/4.0/.

\section{References}

1. Manohar, S., \& Razansky, D. (2016). Photoacoustics: a historical review. Advances in Optics and Photonics, 8(4), 586-617. https://doi.org/10.1364/AOP.8.000586.

2. Ntziachristos, V., Ripoll, J., Wang, L. V., \& Weissleder, R. (2005). Looking and listening to light: the evolution of whole-body photonic imaging. Nature Biotechnology, 23(3), 313-320. https://doi.org/10.1038/nbt1074.

3. Xia, J., Yao, J., \& Wang, L. V. (2014). Photoacoustic tomography: principles and advances. Electromagnetic waves (Cambridge, Mass.), 147, 1-22. https://www.ncbi.nlm.nih.gov/pmc/articles/ PMC4311576/.

4. Emelianov, S., Wang, B., Su, J., Karpiouk, A., Yantsen, E., Sokolov, K, et al. (2008). Intravascular ultrasound and photoacoustic imaging. In Conference proceedings: ... Annual International Conference of the IEEE Engineering in Medicine and Biology Society. IEEE Engineering in Medicine and Biology Society. Annual Conference (pp. 2-5). https://doi.org/10.1109/IEMBS .2008.4649075.

5. Y1, S., Chou, C. Y., Hsieh, B. Y., \& Li, P. C. (2011). Image reconstruction in intravascular photoacoustic imaging. IEEE Transactions on Ultrasonics, Ferroelectrics, and Frequency Control, 58(10), 2067-2077. https://doi.org/10.1109/TUFFC.2011.2057.

6. Xia, J., Wei, C., Pelivanov, I. M., \& O’Donnell, M. (2011). Photoacoustic imaging using narrow beam scanning. In 2011 IEEE International Ultrasonics Symposium (pp. 2380-2383). Presented at the 2011 IEEE International Ultrasonics Symposium. https://doi.org/10.1109/ULTSYM.2011.0591. 
7. Miida, Y., \& Matsuura, Y. (2013). All-optical photoacoustic imaging system using fiber ultrasound probe and hollow optical fiber bundle. Optics Express, 21(19), 22023-22033. https://doi. org/10.1364/OE.21.022023.

8. Colchester, R. J., Zhang, E. Z., Mosse, C. A., Beard, P. C., Papakonstantinou, I., \& Desjardins, A. E. (2015). Broadband miniature optical ultrasound probe for high resolution vascular tissue imaging. Biomedical Optics Express, 6(4), 1502-1511. https://doi.org/10.1364/BOE.6.001502.

9. Chen, S.-L. (2016). Review of laser-generated ultrasound transmitters and their applications to alloptical ultrasound transducers and imaging. Applied Sciences, 7(1), 25. https://doi.org/10.3390/ app7010025.

10. Perekatova, V., Subochev, P., Kleshnin, M., \& Turchin, I. (2016). Optimal wavelengths for optoacoustic measurements of blood oxygen saturation in biological tissues. Biomedical Optics Express, 7(10), 3979-3995. https://doi.org/10.1364/BOE.7.003979.

11. Chee, R. K. W., Zhang, P., Maadi, M., \& Zemp, R. J. (2017). Multifrequency interlaced CMUTs for photoacoustic imaging. IEEE Transactions on Ultrasonics, Ferroelectrics, and Frequency Control, 64(2), 391-401. https://doi.org/10.1109/TUFFC.2016.2620381.

12. Liang, Y., Jin, L., Wang, L., Bai, X., Cheng, L., \& Guan, B.-O. (2017). Fiber-laser-based ultrasound sensor for photoacoustic imaging. Scientific Reports. https://doi.org/10.1038/srep40849.

13. Hu, S., \& Wang, L. V. (2010). Photoacoustic imaging and characterization of the microvasculature. Journal of Biomedical Optics, 15(1), 011101. https://doi.org/10.1117/1.3281673.

14. Wang, B., Su, J. L., Karpiouk, A. B., Sokolov, K. V., Smalling, R. W., \& Emelianov, S. Y. (2010). Intravascular photoacoustic imaging. IEEE Journal of Quantum Electronics, 16(3), 588-599. https ://doi.org/10.1109/JSTQE.2009.2037023.

15. Graf, I. M., Kim, S., \& Emelianov, S. (2010). Staging atherosclerosis using ultrasound, strain and photoacoustic imaging. In 2010 IEEE International Ultrasonics Symposium (pp. 2131-2134). Presented at the 2010 IEEE International Ultrasonics Symposium. https://doi.org/10.1109/ULTSY M.2010.5935831.

16. Xu, G., Meng, Z., Lin, J., Deng, C., Carson, P., \& Fowlkes, J., et al. (2015). In vivo biopsy by photoacousticUS based tissue characterization. In 2015 IEEE International Ultrasonics Symposium (IUS) (pp. 1-4). Presented at the 2015 IEEE International Ultrasonics Symposium (IUS). https://doi. org/10.1109/ULTSYM.2015.0216.

17. Feng, T., Li, Q., Zhang, C., Xu, G., Guo, L. J., Yuan, J., et al. (2016). Characterizing cellular morphology by photoacoustic spectrum analysis with an ultra-broadband optical ultrasonic detector. Optics Express, 24(17), 19853-19862.

18. Cao, Y., Hui, J., Kole, A., Wang, P., Yu, Q., Chen, W., et al. (2016). High-sensitivity intravascular photoacoustic imaging of lipid-laden plaque with a collinear catheter design. Scientific Reports, 6 , srep25236. https://doi.org/10.1038/srep25236.

19. Schwarz, M., Soliman, D., Omar, M., Buehler, A., Ovsepian, S. V., Aguirre, J., et al. (2017). Optoacoustic dermoscopy of the human skin: tuning excitation energy for optimal detection bandwidth with fast and deep imaging in vivo. IEEE Transactions on Medical Imaging, 36(6), 1287-1296. https://doi.org/10.1109/TMI.2017.2664142.

20. Xu, G., Meng, Z.-X., Lin, J.-D., Deng, C. X., Carson, P. L., Fowlkes, J. B., et al. (2016). High resolution physio-chemical tissue analysis: towards non-invasive in vivo biopsy. Scientific Reports, 6, 16937. https://doi.org/10.1038/srep16937.

21. Chitnis, P. V., Mamou, J., McLaughlan, J., Murray, T., \& Roy, R. A. (2009). Photoacoustic thermometry for therapeutic hyperthermia. In 2009 IEEE International Ultrasonics Symposium (pp. 1757-1760). Presented at the 2009 IEEE International Ultrasonics Symposium. https://doi. org/10.1109/ULTSYM.2009.5441585.

22. Wei, C. w, Nguyen, T.-M., Xia, J., Arnal, B., Pelivanov, I., \& O'Donnell, M. (2014). Clinically translatable ultrasound/photoacoustic imaging for real-time needle biopsy guidance. In 2014 IEEE International Ultrasonics Symposium (pp. 839-842). Presented at the 2014 IEEE International Ultrasonics Symposium. https://doi.org/10.1109/ULTSYM.2014.0206.

23. Villanueva-Palero, Y., Hondebrink, E., Petersen, W., \& Steenbergen, W. (2017). Quantitative photoacoustic integrating sphere (QPAIS) platform for absorption coefficient and Grüneisen parameter measurements: Demonstration with human blood. Photoacoustics, 6(Supplement C), 9-15. https:// doi.org/10.1016/j.pacs.2017.03.004.

24. Wei, C.-W., Nguyen, T.-M., Xia, J., Arnal, B., Wong, E. Y., Pelivanov, I. M., et al. (2015). Real-time integrated photoacoustic and ultrasound (PAUS) imaging system to guide interventional procedures: 
ex vivo study. IEEE Transactions on Ultrasonics, Ferroelectrics, and Frequency Control, 62(2), 319-328. https://doi.org/10.1109/TUFFC.2014.006728.

25. Karpiouk, A. B., Aglyamov, S. R., Mallidi, S., Shah, J., Scott, W. G., Rubin, J. M., et al. (2008). Combined ultrasound and photoacoustic imaging to detect and stage deep vein thrombosis: phantom and ex vivo studies. Journal of Biomedical Optics, 13(5), 054061. https://doi.org/10.1117/1.29921 75.

26. Please provide the missing reference [26]

27. Yang, J.-M., Favazza, C., Chen, R., Yao, J., Cai, X., Maslov, K., et al. (2012). Simultaneous functional photoacoustic and ultrasonic endoscopy of internal organs in vivo. Nature Medicine, 18(8), 1297-1302. https://doi.org/10.1038/nm.2823.

28. Bouchard, R., Sahin, O., \& Emelianov, S. (2014). Ultrasound-guided photoacoustic imaging: current state and future development. IEEE Transactions on Ultrasonics, Ferroelectrics, and Frequency Control, 61(3), 450-466. https://doi.org/10.1109/TUFFC.2014.2930.

29. VanderLaan, D., Karpiouk, A. B., Yeager, D., \& Emelianov, S. (2017). Real-time intravascular ultrasound and photoacoustic imaging. IEEE Transactions on Ultrasonics, Ferroelectrics, and Frequency Control, 64(1), 141-149. https://doi.org/10.1109/TUFFC.2016.2640952.

30. Dai, X., Yang, H., Shan, T., Xie, H., Berceli, S. A., \& Jiang, H. (2017). Miniature endoscope for multimodal imaging. ACS Photonics, 4(1), 174-180. https://doi.org/10.1021/acsphotonics.6b00852.

31. Bychkov, A. S., Zarubin, V. P., Karabutov, A. A., Simonova, V. A., \& Cherepetskaya, E. B. (2017). On the use of an optoacoustic and laser ultrasonic imaging system for assessing peripheral intravenous access. Photoacoustics, 5, 10-16. https://doi.org/10.1016/j.pacs.2017.01.002.

32. Ghoshal, G., Mamou, J., \& Oelze, M. L. (2013). State of the art methods for estimating backscatter coefficients. In Quantitative Ultrasound in Soft Tissues (pp. 3-19). New York: Springer. https://link. springer.com/chapter/10.1007/978-94-007-6952-6_1.

33. Sudarshan, V. K., Mookiah, M. R. K., Acharya, U. R., Chandran, V., Molinari, F., Fujita, H., et al. (2016). Application of wavelet techniques for cancer diagnosis using ultrasound images: A review. Computers in Biology and Medicine, 69, 97-111. https://doi.org/10.1016/j.compbiomed .2015.12.006.

34. Beard, P. (2011). Biomedical photoacoustic imaging. Interface Focus, 1(4), 602-631. https://doi. org/10.1098/rsfs.2011.0028.

35. Mallidi, S., Luke, G. P., \& Emelianov, S. (2011). Photoacoustic imaging in cancer detection, diagnosis, and treatment guidance. Trends in Biotechnology, 29(5), 213-221. https://www.sciencedirect. com/science/article/pii/S0167779911000175.

36. Zhang, E., Laufer, J., \& Beard, P. (2008). Backward-mode multiwavelength photoacoustic scanner using a planar Fabry-Perot polymer film ultrasound sensor for high-resolution three-dimensional imaging of biological tissues. Applied Optics, 47(4), 561-577. https://doi.org/10.1364/A0.47.00056 1.

37. Hou, Y., Kim, J.-S., Huang, S.-W., Ashkenazi, S., Guo, L. J., \& O’Donnell, M. (2008). Characterization of a broadband all-optical ultrasound transducer-from optical and acoustical properties to imaging. IEEE Transactions on Ultrasonics, Ferroelectrics, and Frequency Control, 55(8), 18671877. https://doi.org/10.1109/TUFFC.2008.870.

38. Liao, P. L., Wang, P. H., Li, M. L., \& Lu, M. S. C. (2013). A CMOS MEMS capacitive ultrasonic sensor array for three-dimensional photoacoustic imaging. In 2013 IEEE 26th International Conference on Micro Electro Mechanical Systems (MEMS) (pp. 613-616). Presented at the 2013 IEEE 26th International Conference on Micro Electro Mechanical Systems (MEMS). https://doi. org/10.1109/MEMSYS.2013.6474316.

39. Hsieh, B.-Y., Chen, S.-L., Ling, T., Guo, L. J., \& Li, P.-C. (2014). All-optical scanhead for ultrasound and photoacoustic imaging -imaging mode switching by dichroic filtering. Photoacoustics, 2(1), 39-46. https://www.sciencedirect.com/science/article/pii/S2213597914000032

40. Hsieh, B.-Y., Chen, S.-L., Ling, T., Guo, L. J., \& Li, P. C. (2011). All-optical generation and detection of acoustic waves for intravascular ultrasound and photoacoustic imaging. In 2011 IEEE International Ultrasonics Symposium (pp. 1175-1178). Presented at the 2011 IEEE International Ultrasonics Symposium. https://doi.org/10.1109/ULTSYM.2011.0289.

41. Zhang, C., Ling, T., Chen, S.-L., \& Guo, L. J. (2014). Ultrabroad bandwidth and highly sensitive optical ultrasonic detector for photoacoustic imaging. ACS Photonics, 1(11), 1093-1098. https://doi. org/10.1021/ph500159g. 
42. Zhang, C., Chen, S. L., Ling, T., \& Guo, L. J. (2015). Review of imprinted polymer microrings as ultrasound detectors: design, fabrication, and characterization. IEEE Sensors Journal, 15(6), 32413248. https://doi.org/10.1109/JSEN.2015.2421519.

43. Zou, X., Wu, N., Tian, Y., \& Wang, X. (2014). Broadband miniature fiber optic ultrasound generator. Optics Express, 22(15), 18119-18127. https://www.osapublishing.org/abstract. cfm?uri=oe-22-15-18119

44. Wissmeyer, G., Pleitez, M. A., Rosenthal, A., \& Ntziachristos, V. (2018). Looking at sound: optoacoustics with all-optical ultrasound detection. Light: Science and Applications. https://doi. org/10.1038/s41377-018-0036-7.

45. Acquafresca, A., Biagi, E., Masotti, L., \& Menichelli, D. (2003). Toward virtual biopsy through an all fiber optic ultrasonic miniaturized transducer: A proposal. IEEE Transactions on Ultrasonics, Ferroelectrics, and Frequency Control, 50(10), 1325-1335. https://doi.org/10.1109/TUFFC .2003.1244749.

46. Biagi, E., Cerbai, S., Masotti, L., Belsito, L., Roncaglia, A., Masetti, G., \& Speciale, N. (2011). Fiber optic broadband ultrasonic probe for virtual biopsy: Technological solutions. Lecture Notes in Electrical Engineering, 91 LNEE, 223-227. https://doi.org/10.1007/978-94-007-1324-6_34.

47. Belsito, L., Vannacci, E., Mancarella, F., Ferri, M., Veronese, G., Biagi, E., et al. (2014). Fabrication of fiber-optic broadband ultrasound emitters by micro-opto-mechanical technology. Journal of Micromechanics and Microengineering. https://doi.org/10.1088/0960-1317/24/8/085003.

48. Vannacci, E., Belsito, L., Mancarella, F., Ferri, M., Veronese, G. P., Roncaglia, A., et al. (2014). Miniaturized fiber-optic ultrasound probes for endoscopic tissue analysis by micro-opto-mechanical technology. Biomedical Microdevices, 16(3), 415-426. https://doi.org/10.1007/s10544-014-9844-6.

49. Vannacci, E., Granchi, S., Biagi, E., Belsito, L., \& Roncaglia, A. (2015). High resolution ultrasonic images by miniaturized fiber-optic probe. Lecture Notes in Electrical Engineering, 319, 349-353. https://doi.org/10.1007/978-3-319-09617-9_61.

50. Vannacci, E., Granchi, S., Belsito, L., Roncaglia, A., \& Biagi, E. (2017). Wide bandwidth fiberoptic ultrasound probe in MOMS technology: preliminary signal processing results. Ultrasonics, 75 , 164-173. https://doi.org/10.1016/j.ultras.2016.11.024.

51. Ustun, A., \& Zou, J. (2018). A novel photoacoustic sensing probe using optical fiber acoustic delay line (vol. 10494). In Presented at the Progress in Biomedical Optics and Imaging-Proceedings of SPIE. https://doi.org/10.1117/12.2288809.

52. Hysi, E., Dopsa, D., \& Kolios, M. C. (2013). Photoacoustic radio-frequency spectroscopy (PA-RFS): A technique for monitoring absorber size and concentration (Vol. 8581). In Presented at the Progress in Biomedical Optics and Imaging-Proceedings of SPIE. https://doi.org/10.1117/12.20049 91.

53. Granchi, S., Vannacci, E., Biagi, E., \& Masotti, L. (2015). Differentiation of breast lesions by use of HyperSPACE: hyper-spectral analysis for characterization in echography. Ultrasound in Medicine \& Biology, 41(7), 1967-1980. https://doi.org/10.1016/j.ultrasmedbio.2015.02.014.

54. Granchi, S., Vannacci, E., Biagi, E., \& Masotti, L. (2016). Multidimensional spectral analysis of the ultrasonic radiofrequency signal for characterization of media. Ultrasonics, 68, 89-101. https://doi. org/10.1016/j.ultras.2016.02.010.

55. Granchi, S., Vannacci, E., \& Biagi, E. (2017). Characterization of benign thyroid nodules with HyperSPACE (Hyper Spectral Analysis for Characterization in Echography) before and after percutaneous laser ablation: a pilot study. Medical Ultrasonography, 19(2), 172. https://doi.org/10.11152/ mu-1039.

56. Mamou, J., \& Oelze, M. L. (Eds.). (2013). Quantitative Ultrasound in Soft Tissues. Amsterdam: Springer. https://www.springer.com/la/book/9789400769519.

57. Madsen, S. J., Patterson, M. S., \& Wilson, B. C. (1992). The use of India ink as an optical absorber in tissue-simulating phantoms. Physics in Medicine and Biology, 37(4), 985-993.

58. Wang, S., Tao, C., Yang, Y., Wang, X., \& Liu, X. (2015). Theoretical and experimental study of spectral characteristics of the photoacoustic signal from stochastically distributed particles. IEEE Transactions on Ultrasonics, Ferroelectrics, and Frequency Control, 62(7), 1245-1255. https://doi. org/10.1109/TUFFC.2014.006806.

Publisher's Note Springer Nature remains neutral with regard to jurisdictional claims in published maps and institutional affiliations. 